\title{
Améliorer l'étiquetage nutritionnel : une cause utile à soutenir !
}

\author{
M. Pigeyre $\cdot$ L. Genser $\cdot$ J. Dargent \\ (C) Lavoisier SAS 2017
}

L'information nutritionnelle figurant sur les produits se doit d'être améliorée afin de fournir aux consommateurs, lors de leurs achats, une information adaptée et de permettre à tous d'avoir une alimentation plus saine et équilibrée.

Depuis mars 2015, une grande concertation a été conduite avec des représentants des industriels, des distributeurs, des consommateurs, des patients, des autorités sanitaires et des scientifiques pour déterminer les différents systèmes graphiques possibles. Quatre logos ont été proposés suivant les deux approches existantes aujourd'hui : l'approche analytique, qui présente les principaux composants du produit et les quantifie (Nutri-Repère et Traffic Lights), et l'approche synthétique, fondée sur un code couleur (NutriScore et SENS). Un premier travail de l'équipe du Pr Hercberg, sur le « Système d'information nutritionnelle à cinq couleurs sur la face avant des emballages : application, performance et perception dans le contexte français » a d'ailleurs été publié dans Obésité (Julia et al, 2015).

Pour répondre à cet objectif, la loi du 26 janvier 2016 relative à la modernisation du système de santé recommande, toutefois « de façon facultative », aux producteurs d'aliments un système d'étiquetage nutritionnel graphique en face avant des produits, pour faciliter le choix d'achat du consommateur au regard de la composition nutritionnelle des produits. Ce système devrait voir le jour en 2017.

Pour tester ces logos auprès des consommateurs, une expérimentation a été conduite en conditions réelles d'achat dans plusieurs grandes surfaces, de septembre à décembre 2016, avec pour objectif de déterminer avec précision le système le plus efficace sur les comportements d'achat. Le rapport complet devrait prochainement être présenté (source : social-santé.gouv.fr).

Toutefois, le 14 février dernier, l'Anses a publié son avis relatif à l'analyse de la pertinence en matière de nutrition de systèmes d'information nutritionnelle (SIN) destinés aux

\footnotetext{
M. Pigeyre $(\square)$

Service de nutrition,

centre hospitalo-universitaire de Lille,

59037 Lille Cedex, France

e-mail : marie.pigeyre-2@univ-lille2.fr
}

consommateurs. Ce travail a consisté à évaluer l'impact potentiel de la mise en œuvre de tels systèmes sur la réduction de l'incidence de certaines pathologies dans l'ensemble de la population, par l'intermédiaire de ses effets sur les choix alimentaires. L'Agence a conclu qu'en l'état actuel des connaissances, la pertinence nutritionnelle dans une perspective de santé publique des SIN examinés n'est pas démontrée (source : Anses.fr).

Ce rapport a été vivement contesté par de nombreux experts et scientifiques, dont la Société française de santé publique, qui dénoncent l'impartialité de l'Anses et ses liens financiers avec l'industrie agroalimentaire, ainsi que la mise à l'écart de certains experts du groupe de travail en raison de liens d'intérêt «professionnels" (source : Le Monde, 28 février 2017). Les scientifiques soulignent également le non-sens des conclusions de l'Agence, rappelant l'impossibilité de démontrer un effet des logos sur l'incidence des maladies chroniques, alors qu'ils ne sont pas encore utilisés.

La bataille sur la pertinence de l'étiquetage nutritionnel est donc loin d'être terminée et, pour argumenter ce débat, vous trouverez dans ce numéro d'Obésité les résultats des travaux menés par l'équipe du Pr S. Hercberg, sur la « Perception de différents systèmes d'information nutritionnelle actuellement proposés en France en fonction du statut pondéral ». Nous tenions par ailleurs à ajouter que le comité éditorial de la revue soutient avec convictions le combat mené par l'équipe du Pr S. Hercberg, sur l'utilité $\mathrm{du}$ NutriScore en face avant des produits, comme signalétique nutritionnelle utile, facilement compréhensible et non stigmatisante.

Dans ce numéro, vous trouverez également l'excellent et exhaustif article de O. Ziegler et al, « De la dysfonction $\mathrm{du}$ tissu adipeux blanc aux phénotypes anatomocliniques de l'obésité »; les résumés des communications présentées lors de la Journée annuelle Benjamin Delessert en février sur les thèmes : « Troubles du comportement alimentaire : nouveaux concepts, nouvelles approches » et «Évictions alimentaires : de l'allergie au mode de vie »; sans oublier l'excellente synthèse des travaux du Pr K. Clément qui a reçu le prix Benjamin Delessert. Du coté de la chirurgie bariatrique, $\mathrm{P}$. Blanc et $\mathrm{C}$. Breton nous présentent un article 
« technique » sur la laparoscopie. Enfin, le J. Dargent nous fait part de son opinion sur « le gaspillage des ressources naturelles, l'épidémie d'obésité, et la pertinence de la chirurgie bariatrique » et ses notes de lecture sur Les métamorphoses du gras. Histoire de l'obésité de Georges Vigarello.
Vous retrouverez, pour finir, une revue de presse des articles incontournables alimentée par notre habituel duo de chirurgiens (L. Genser et C. Barrat) et un nouveau duo médecin-psychologue (V. Florent et B. Gaudrat) qui vient de nous rejoindre.

Bonne lecture ! 